\title{
PESQUISA DE Staphylococcus aureus RESISTENTE À METICILINA (MRSA) EM METRÔS DA REGIÃO METROPOLITANA DO RECIFE-PE
}

DOI: 10.22289/2446-922X.V5N2A3

\author{
Paulo Ricardo Anjos do Monte \\ Maria Amélia Vieira Maciel \\ Laury Francis Costa \\ Jailton Lobo da Costa Lima ${ }^{1}$
}

\section{RESUMO}

A disseminação de micro-organismos multirresistentes tem se tornado um problema de saúde pública, e os meios de transporte são uma via de contaminação por estes micro-organismos. O objetivo deste trabalho foi identificar a ocorrência de Staphylococcus aureus resistente à meticilina (MRSA) contaminando vagões dos metrôs que circulam pela Região Metropolitana do Recife- PE. Realizou-se a coleta de 16 a 18 de abril de 2018 numa área de $24 \mathrm{~cm}^{2}$, com "swab", de 45 amostras dos vagões. De cada vagão foram coletadas 5 amostras dos seguintes locais: janela, corrimão alto, assento, porta e corrimão baixo. Foram analisadas as três linhas: Jaboatão, Camaragibe, e Cajueiro seco (sul). Foi realizada a identificação dos micro-organismos através de testes bioquímicos manuais e a análise da susceptibilidade foi realizada pelo método de disco difusão comos antibióticos utilizados foram Clindamicina, Eritromicina, Cefoxitina, Sulfazotrim, Gentamicina, Clorafenicol, Tetraciclina e Ciprofloxacina. De quarenta e cinco amostras coletadas em nove metrôs, três de cada linha, vinte e nove $(64,44 \%)$ foram positivas para $S$. aureus. Destas amostras positivas, 13 (44,82\%) foram do metrô da linha Jaboatão, seguido da linha Camaragibe e linha Cajueiro Seco (Sul) com oito (27,59\%) amostras cada. Todas as amostras foram sensíveis a cefoxitina. Este estudo não identificou a ocorrência de MRSA como contaminantes nos vagões do metrô que circulam pela Região Metropolitana do Recife- PE, mas identificou o crescente aumento da resistência de $S$. aureus a eritromicina e a clindamicina, sugerindo desta forma a necessidade de higienização dos metrôs visando a prevenção de contaminação dos usuários.

Palavras-chave: Staphylococcus aureus resistente à meticilina; Contaminação; Metrôs.

\section{ABSTRACT}

The spread of multiresistant microorganisms has become a public health problem, and the means of transportation are a pathway of contamination by these microorganisms. The objective of this study was to identify the occurrence of methicillin-resistant Staphylococcus aureus (MRSA)

\footnotetext{
${ }^{1}$ Endereço eletrônico de contato: jailtonlobo@hotmail.com

Recebido em 07/11/2019. Aprovado pelo Conselho Editorial e aceito para publicação em 10/12/2019.

Rev. Psicol Saúde e Debate. Dez., 2019:5(2):43-51
} 
contaminating wagons of the subways that circulate in the Metropolitan Region of Recife-PE. The collection was carried out from April 16 to 18, 2018 in an area of $24 \mathrm{~cm}^{2}$, with swab, of 45 samples of the wagons. From each car, 5 samples were collected from the following locations: window, high handrail, seat, door and low handrail. The three lines were analyzed: Jaboatão, Camaragibe, and Cajueiro seco (south). The microorganisms were identified through manual biochemical tests and the susceptibility analysis was performed by the disc diffusion method. The antibiotics used were Clindamycin, Erythromycin, Cefoxitin, Sulfazotrim, Gentamicin, Chlorphenicol, Tetracycline and Ciprofloxacin. Of forty - five samples collected in nine subways, three of each line, twenty nine $(64.44 \%)$ were positive for S. aureus. Of these positive samples, $13(44.82 \%)$ were from the subway of the Jaboatão line, followed by the Camaragibe line and the Cajueiro Seco (South) line with eight $(27.59 \%)$ samples each. All samples were sensitive to cefoxitin. This study did not identify the occurrence of MRSA as contaminants in the subway cars that circulate in the Metropolitan Region of Recife, but identified the increasing increase of S. aureus resistance to erythromycin and clindamycin, thus suggesting the need for hygienization of the subways aiming at the prevention of contamination of users.

Keywords: Methicillin-resistant Staphylococcus aureus; Contamination; Subways.

\section{INTRODUÇÃO}

As bactérias podem ser um problema crítico para a saúde da população pela facilidade de exposição dos usuários do sistema de transporte público a esses patógenos (Simões et al., 2011). Para que uma superfície inerte, como as de um ônibus ou metrô, possa servir de via de contaminação, deve existir interação entre pessoas e a superfície (Stuchi, Oliveira, Soares, \& Arreguy-Sena, 2013). A má higienização das mãos dos usuários dos transportes públicos pode servir como fonte de contaminação para outros indivíduos por meio do contato das mãos contaminadas com as superfícies dos transportes (Stuchi et al., 2013).

Em estudo realizado na cidade de São Paulo foi avaliado o potencial infeccioso do transporte público de passageiros, onde se evidenciou a presença de vários microrganismos, entre eles Micrococcus sp., Burkholderia mallei, Bacillus subtilis, Acinetobacter baumanii, Staphylococcus coagulase negativo e Staphylococcus aureus (Mendonça, Olival, Mímica, Navarini, Paschoalotti, \& Chieffi, 2008). S. aureus são bactérias Gram positivas encontradas na microbiota humana, que atuam na defesa contra infecções por outros patógenos. No entanto esta bactéria pode colonizar a superfície de diferentes objetos como maçanetas, telefones, superfícies de ônibus e metrôs, por encontrar condições favoráveis para sobreviver, permanecendo por longos períodos, podendo desencadear doenças infecciosas via contato com indivíduos suscetíveis (Evangelista \& Oliveira, 2015; Santiago-Neto, 2014; Pinheiro \& Stopiglia, 2015).

A contaminação de usuários dos transportes públicos por $S$. aureus pode causar desde simples infecções como espinhas, furúnculos e celulites, a até graves infecções que é o caso de septicemia, endocardite, síndrome do choque tóxico, pneumonia, meningite e outras (Santos et al., 2007). Tornando, portanto, a contaminação por este microrganismo preocupante, uma vez 
que o mesmo pode apresentar resistência para os antibióticos beta-lactâmicos, a classe de antibióticos mais prescrita nos hospitais do Brasil (França, 2012).

O principal mecanismo de resistência de $S$. aureus a estes antibióticos beta-lactâmicos está relacionado com a mutação da proteína PBP (proteína ligadora de penicilina), denominado de MRSA (Staphylococcus aureus resistente à meticilina) (Tavares, 2000). O MRSA, é um dos patógenos de maior relevância na saúde pública, por ocasionar alta taxa de morbidade e mortalidade em pacientes hospitalizados (Simões et al., 2011).

Considerando a exposição da população diariamente nos vagões do metrô a vários agentes patogênicos, dentre eles o MRSA, este estudo teve como objetivo identificar a ocorrência de MRSA como contaminantes nos vagões dos metrôs que circulam pela Região Metropolitana do Recife- PE.

\section{METODOLOGIA}

\section{Tipo de estudo}

Trata-se de uma pesquisa experimental, descritiva com abordagem quantitativa, realizada nos metrôs da cidade do Recife, nas linhas Sul (Cajueiro Seco), Camaragibe e Jaboatão, para identificação de contaminação por MRSA.

\section{Coleta e identificação dos isolados}

As coletas foram realizadas em três dias consecutivos, 16, 17 e 18, do mês de abril de 2018, em nove metrôs diferentes, sendo três da linha Camaragibe, três de Jaboatão e três de Cajueiro seco (Sul). Os locais das coletas foram selecionados estrategicamente, procurando determinar assim os pontos de intenso contato dos usuários e maior viabilidade de contaminação, como corrimão alto e baixo, porta, janela e assentos todos tocados superficialmente pelos usuários.

Para a coleta das amostras, utilizou-se a técnica de Swab estéril. Cada área foi delimitada por um quadrante com área de $24 \mathrm{~cm}^{2}$ sendo a coleta realizada por meio de esfregaço superficial, obtendo cinco amostras de cada um dos noves metrôs analisados. Os "swabs" previamente identificados com o código da linha do metrô e o local da coleta foram colocados em meio Cary-Blair e transportados em uma caixa de isopor e encaminhados para o Laboratório de Bacteriologia e Biologia Molecular da Universidade Federal de Pernambuco para análise.

As amostras foram semeadas e depois inoculados em ágar sangue de carneiro a $5 \% \mathrm{e}$ incubados por $24-48$ horas a $37^{\circ} \mathrm{C}$. As colônias com características macroscópicas do gênero Staphylococcus foram coradas com Gram, quando confirmadas pela morfologia e coloração, 
foram submetidas à identificação pelos testes: catalase, coagulase, fermentação do manitol e desoxirribonuclease (DNase) (Winn et al., 2008).

\section{Perfil de susceptibilidade}

Após a identificação, o teste de suscetibilidade de $S$. aureus foi realizado pelo método de

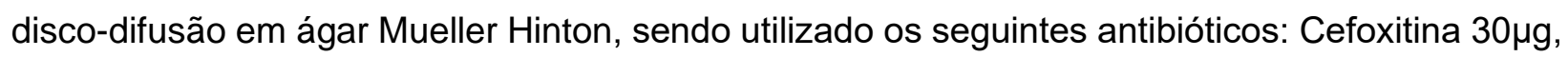
Gentamicina $10 \mu \mathrm{g}$, Tetraciclina $30 \mu \mathrm{g}$, Eritromicina $15 \mu \mathrm{g}$, Ciprofloxacina $5 \mu \mathrm{g}$, Clindamicina $2 \mu \mathrm{g}$, Sulfazotrim $300 \mu \mathrm{g}$ e Cloranfenicol $30 \mu \mathrm{g}$. Foram considerados os critérios de interpretação do Clinical and Laboratory Standards Institute (CLSI, 2018).

\section{Teste D}

Isolados de $S$. aureus com resistência à eritromicina e suscetibilidade ou resistência intermediária à clindamicina no antibiograma foram selecionados. O teste foi realizado conforme proposto por Pereira et al. (2016). O teste D (resistência a macrolídeos, lincosaminas e estreptogramina) analisa o efeito antagônico gerado entre a associação da eritromicina com a clindamicina, os isolados que não mostraram achatamento da inibição da zona ao redor do disco de clindamicina foram relatados como susceptíveis a clindamicina (teste $D$ negativo) e isolados que mostraram achatamento da zona de inibição em torno do disco de clindamicina adjacente ao disco de eritromicina (zona "D") indica indução a resistência à clindamicina (teste D positivo) (Otter \& French, 2009; Fernandes, Rangel, Sena, Rangel \& Moraes, 2012).

Os dados coletados foram digitados e organizados no programa Excel® 2007, para serem analisados.

\section{RESULTADOS}

\section{Coleta e identificação dos isolados}

De 45 amostras coletadas em nove metrôs, três de cada linha, 29 (64,44\%) foram positivas para S. aureus. Destas amostras positivas, $13(44,82 \%)$ foram do metrô da linha Jaboatão, seguido da linha Camaragibe e linha Sul com oito $(27,59 \%)$ amostras cada. A taxa de contaminação das áreas coletadas foram: janelas e corrimão baixo com sete $(24,14 \%)$ amostras cada, corrimão alto, assento e porta com cinco (17,24\%) amostras, como observado na Tabela 1 . 
Tabela 1. Número de amostras positivas para $S$. aureus por local de coleta por linha de metrô.

\begin{tabular}{lcccccc}
\hline \multicolumn{1}{c}{ Linha } & \multicolumn{7}{c}{ Local } \\
\cline { 2 - 7 } & Janela & Porta & Corrimão Alto & Assento & Corrimão Baixo & Total \\
Camaragibe & 2 & 1 & 2 & 0 & 3 & 8 \\
Sul & 2 & 2 & 1 & 2 & 1 & 8 \\
Jaboatão & 3 & 2 & 2 & 3 & 3 & 13 \\
Total & 7 & 5 & 5 & 5 & 8 & 29 \\
\hline
\end{tabular}

\section{Perfil de susceptibilidade}

Foi analisado o perfil de susceptibilidade dos 29 isolados de $S$. aureus, não tendo sido encontrado nenhum MRSA nas amostras analisadas, uma vez que não houve nenhuma amostra resistente a Cefoxitina, que junto com a Gentamicina foram os antibióticos mais eficazes, apresentando 100\% de eficácia frente aos S. aureus analisados, seguidos da Ciprofloxaina que teve eficácia de $96,5 \%$. O antibiótico que apresentou menor eficácia frente aos isolados de $S$. aureus desta pesquisa foi a Eritromicina, com $37,93 \%$ de sensibilidade, como pode ser observado na Tabela 2.

Tabela 2. Perfil de susceptibilidade de $S$. aureus provenientes de metrôs da região metropolitana do Recife-PE

\begin{tabular}{|c|c|c|c|c|c|c|c|c|}
\hline \multirow[t]{2}{*}{ Amostra(no) } & \multicolumn{8}{|c|}{ Antibiótico testado } \\
\hline & CFO & SUF & CLO & TET & CIP & GEN & ERI & CLI \\
\hline JAB (1) & $\mathrm{S}$ & $\mathrm{s}$ & S & $\mathrm{s}$ & $\mathrm{s}$ & $\mathrm{s}$ & $\mathrm{S}$ & $\mathrm{S}$ \\
\hline JAB (2) & $S$ & $S$ & $S$ & $S$ & $S$ & $S$ & $S$ & $S$ \\
\hline JAB (3) & $\mathrm{s}$ & $\mathrm{s}$ & $\mathrm{s}$ & $\mathrm{s}$ & $S$ & $\mathrm{~s}$ & $S$ & S \\
\hline JAB (4) & $S$ & $S$ & $S$ & $S$ & $S$ & $S$ & 1 & $S$ \\
\hline SUL (5) & $\mathrm{s}$ & $\mathrm{s}$ & $\mathrm{s}$ & $\mathrm{s}$ & $\mathrm{s}$ & $\mathrm{s}$ & $\mathrm{s}$ & $\mathrm{s}$ \\
\hline SUL (6) & $S$ & $S$ & $S$ & $S$ & $S$ & $S$ & $\mathrm{R}$ & $S$ \\
\hline SUL (7) & $\mathrm{s}$ & $\mathrm{R}$ & $\mathrm{s}$ & $\mathrm{s}$ & $\mathrm{s}$ & $\mathrm{s}$ & $\mathrm{s}$ & $\mathrm{s}$ \\
\hline SUL (8) & $S$ & $S$ & $S$ & $\mathrm{R}$ & $S$ & $S$ & $\mathrm{R}$ & $R$ \\
\hline GIB (9) & $\mathrm{s}$ & $\mathrm{R}$ & $\mathrm{s}$ & $\mathrm{s}$ & $\mathrm{s}$ & $\mathrm{s}$ & $\mathrm{R}$ & $\mathrm{s}$ \\
\hline JAB (10) & $S$ & $S$ & $S$ & $S$ & $S$ & $S$ & $\mathrm{R}$ & $\mathrm{R}$ \\
\hline GIB (11) & $\mathrm{s}$ & $\mathrm{s}$ & $\mathrm{s}$ & $s$ & $\mathrm{~s}$ & $\mathrm{~s}$ & $\mathrm{R}$ & $\mathrm{s}$ \\
\hline GIB (12) & $S$ & $S$ & $S$ & $S$ & $S$ & $S$ & $\mathrm{R}$ & S \\
\hline JAB (13) & $\mathrm{s}$ & $\mathrm{s}$ & $\mathrm{s}$ & $\mathrm{s}$ & $\mathrm{s}$ & $\mathrm{s}$ & $\mathrm{s}$ & $\mathrm{s}$ \\
\hline JAB (14) & $\mathrm{s}$ & $\mathrm{s}$ & $\mathrm{s}$ & $\mathrm{s}$ & $S$ & $\mathrm{~s}$ & $\mathrm{R}$ & $\mathrm{R}$ \\
\hline
\end{tabular}




\begin{tabular}{|c|c|c|c|c|c|c|c|c|}
\hline GIB (15) & $S$ & $\mathrm{~S}$ & $S$ & $S$ & $\mathrm{~S}$ & $S$ & I & $\mathrm{R}$ \\
\hline JAB (16) & $S$ & $S$ & $S$ & $S$ & $S$ & $S$ & $S$ & S \\
\hline SUL (17) & $S$ & $S$ & $S$ & $S$ & $S$ & $S$ & $S$ & $S$ \\
\hline SUL (18) & $\mathrm{s}$ & $\mathrm{s}$ & S & $\mathrm{s}$ & $\mathrm{S}$ & $S$ & $\mathrm{R}$ & $\mathrm{R}$ \\
\hline SUL (19) & $S$ & $S$ & $S$ & $S$ & $S$ & $S$ & $\mathrm{R}$ & $S$ \\
\hline SUL (20) & $S$ & $S$ & $\mathrm{R}$ & $S$ & $\mathrm{R}$ & $S$ & $\mathrm{R}$ & $\mathrm{R}$ \\
\hline GIB (21) & $S$ & $S$ & $S$ & $S$ & $S$ & $S$ & I & $S$ \\
\hline GIB (22) & $\mathrm{s}$ & $\mathrm{s}$ & $\mathrm{R}$ & $\mathrm{s}$ & $\mathrm{S}$ & $S$ & $\mathrm{R}$ & $\mathrm{S}$ \\
\hline JAB (23) & $S$ & $S$ & $S$ & $\mathrm{R}$ & $S$ & $S$ & $\mathrm{R}$ & $S$ \\
\hline JAB (24) & $S$ & $S$ & $S$ & $S$ & $S$ & $S$ & $S$ & $\mathrm{~s}$ \\
\hline GIB (25) & $S$ & $S$ & $S$ & $S$ & $S$ & $S$ & $\mathrm{R}$ & I \\
\hline GIB (26) & $\mathrm{s}$ & $\mathrm{s}$ & $S$ & $\mathrm{~s}$ & $\mathrm{~s}$ & $S$ & $\mathrm{R}$ & $\mathrm{s}$ \\
\hline JAB (27) & S & $\mathrm{s}$ & S & $\mathrm{s}$ & $\mathrm{s}$ & $S$ & S & $\mathrm{S}$ \\
\hline JAB (28) & $\mathrm{s}$ & $\mathrm{R}$ & S & $\mathrm{s}$ & $\mathrm{s}$ & S & $\mathrm{R}$ & $\mathrm{R}$ \\
\hline JAB (29) & S & $\mathrm{s}$ & S & $\mathrm{s}$ & $\mathrm{s}$ & $S$ & S & $\mathrm{S}$ \\
\hline
\end{tabular}

gentamicina; ERI: eritromicina; CLI: clindamicina; S: sensível; I: Resistência intermediária; R: resistente.

\section{Teste D}

Neste trabalho, quando foi analisado o teste D, foram detectadas três amostras positivas (10,34\%), tendo elas apresentando o fenótipo $\mathrm{MLS}_{\mathrm{B}}$, cujo mecanismo de resistência está associado a uma alteração ribossômica (gene erm), todas as amostras positivas foram oriundas da linha Jaboatão. Na Figura1 podemos observar o achatamento da zona de inibição em torno do disco de clindamicina adjacente ao disco de eritromicina (zona "D") indicando indução a resistência à clindamicina. O presente estudo foi uns dos poucos a analisar este tipo de teste para amostras de contaminação de ambientes. servindo de alerta por limitar as opções terapêuticas frente a estas bactérias. 
Figura 1 Teste D positivo na amostra 10 coletada em assento da linha Jaboatão.

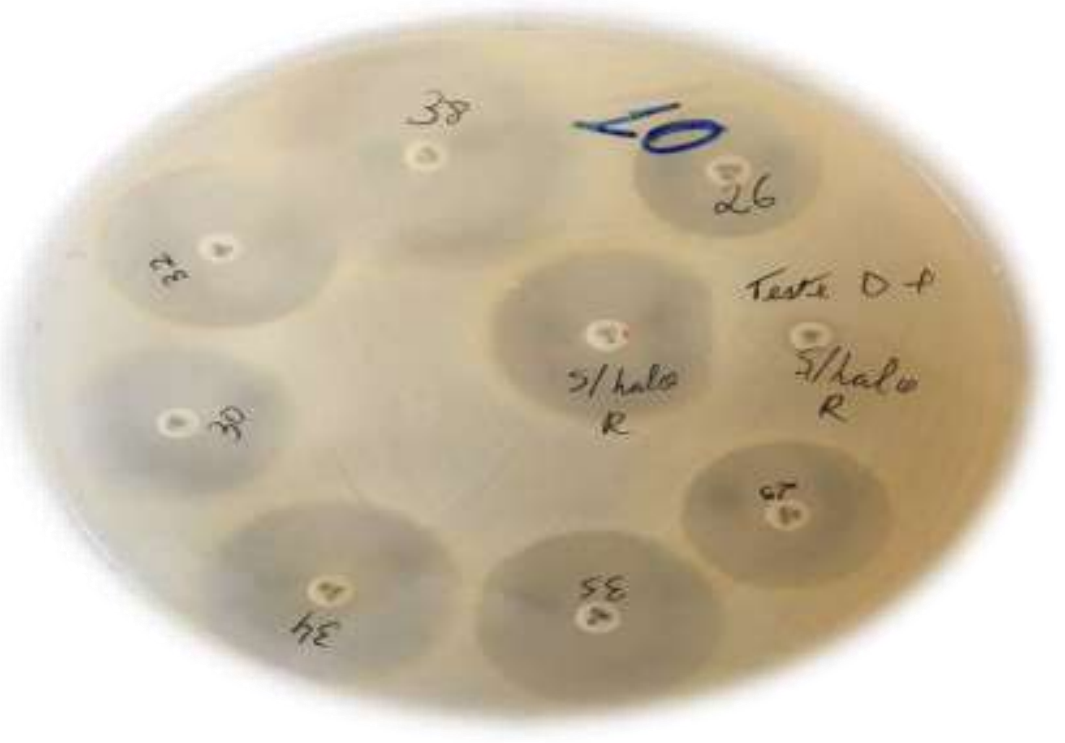

\section{DISCUSSÃO}

Este trabalho verificou que 29 (64,44\%) das amostras foram positivas para $S$. aureus no ambiente do metrô, sendo considerado um número elevado de amostras positivas se comparado ao número total de amostras coletadas, 45 amostras. Diferindo assim de outros estudos, como o trabalho descrito por Mendonça et al. (2008), em que analisaram a contaminação em ônibus do sistema de transporte público da cidade de São Paulo, onde das 120 amostras coletadas apenas sete $(4,79 \%)$ foram positivas para $S$. aureus. Assim como no estudo de Otter \& French (2009), em que foram analisadas amostras de ônibus, metrôs, estações de metrôs, hotéis e áreas públicas de um hospital no centro de Londres-Inglaterra, das 118 amostras analisadas apenas nove (8\%) foram positivas para o S. aureus. Além do que foi constatado por Yeh, Simon, Millar, Alexander \& Franklin (2011), em amostras obtidas em ônibus do sistema de transporte público de Portland-EUA, dentre as 70 amostras coletadas apenas $14(20 \%)$ foram positivas para $S$. aureus.

Em estudo similar realizado por Peng et al. (2015) na China, analisando 320 amostras coletadas nos metrôs da cidade do Cantão, oito (2,5\%) delas foram positivas para MRSA. No estudo realizado por Simões et al. (2011), na cidade do Porto, em Portugal, foi verificado que de 85 amostras colhidas de ônibus, foram encontradas 22 (26\%) amostras positivas para MRSA. Enquanto o estudo de Conceição, Diamantino, Coelho, Lencastre, Aires-de-Sousa (2013), em Lisboa, das 199 amostras coletadas em ônibus, 72 (36,2\%) foram positivas para MRSA. 
Estudos disponíveis sobre a prevalência, características e fenótipos do $S$. aureus no sistema de metrô de todo o mundo, são limitados e não abordam o perfil de resistência desses isolados a outros antimicrobianos.

A resistência a Eritromicina e a Clindamicina, antibióticos das classes dos macrolídeos e lincosaminas respectivamente, é caracterizada pelo fenótipo erm. Estes antibióticos são utilizados como alternativa para o tratamento de pacientes alérgicos a penicilina em casos de infecções de pele e em tecidos moles por $S$. aureus, e a resistência a estes antibióticos tem se mostrado muito comum e com isso a importância do rastreio desses fenótipos de resistência visando combater e evitar a multirresistência de S. aureus (Noel, Silvério, Francisco, Almeida \& Soares ,2017; Fracarolli, Oliveira, \& Marziale,2017).

\section{CONSIDERAÇÕES}

Apesar do presente estudo não ter encontrado a presença de MRSA em nenhuma das amostras, isso não diminui o potencial infeccioso que existe nos vagões do metrô da região metropolitana do Recife-PE, uma vez que foi detectado outro fenótipo de resistência a outras classes e antibióticos que são utilizadas para o tratamento de infecções por $S$. aureus, demonstrando assim a importância do rastreio desses fenótipos de resistência visando combater e evitar a disseminação de cepas de $S$. aureus com multirresistência.

\section{REFERÊNCIAS}

Clinical and Laboratory Standards Institute (2018). Performance standards for antimicrobial susceptibility testing; twenty-eight informational supplement, M100-S27. Wayne, PA: CLSI.

Conceição, T., Diamantino, F., Coelho, C., Lencastre, H. \& Aires-de-Sousa, M. (2013). Contamination of Public Buses with MRSA in Lisbon, Portugal: A Possible Transmission Route of Major MRSA Clones within the Community. PLOS ONE, 8(11). https://doi.org/10.1371/journal.pone.0077812

Evangelista, S. S., \& Oliveira, A. C. (2015). Staphylococcus aureus meticilino resistente adquirido na comunidade: um problema mundial. Rev Bras Enferm, 68(1), 136-143. http://dx.doi.org/10.1590/0034-7167.2015680119p

Fernandes, A. A. L., Rangel, C. D., Sena, C. J. C., Rangel, C. V. \& Moraes, R. (2012). Diversidade de bactérias, fungos e formas de resistência de parasitos em duas rotas de ônibus do transporte coletivo da Grande Vitória-ES. Revista Sapientia, 11, 39-45.

Fracarolli, I. F. L., Oliveira, S. A., \& Marziale, M. H. P. (2017). Colonização bacteriana e resistência antimicrobiana em trabalhadores de saúde: revisão integrativa. Acta Paulista de Enfermagem, 30(6), 651-657. https://dx.doi.org/10.1590/1982-0194201700086

França, L.C. (2012). Principais antibióticos utilizados em hospitais brasileiros nos últimos 10 anos. Brasil: Monografia. Universidade Federal de Santa Catarina. Centro de Ciências da Saúde Pública. XIV Curso de Especialização em Saúde Pública. 
M. R. I. et al. (2014). Relação da idade na presença de bactérias resistentes a antimicrobianos em rebanhos leiteiros no Rio Grande do Sul. Pesq. Vet. Bras, Rio de Janeiro, 34(7), 613620.

Mendonça, R. G. M., Olival, G. S., Mímica, L. M. J., Navarini, A., Paschoalotti, M. A., \& Chieffi, P. P. (2008). Potencial infeccioso do transporte público de passageiros da cidade de São Paulo. Arq Med Hosp Fac Cienc Med Santa Casa São Paulo, 53, 53-57.

Noel, C. C., Silvério, F. M., Francisco, N. L. S. G., Almeida, N. R. A. \& Soares, L. C. (2017). Suscetibilidade antimicrobiana e fatores de virulência de Staphylococcus em fômites do hospital universitário sul fluminense. Rev. Bras. Cien. Saúde., Rio de Janeiro, 21(3), 245254. https://doi.org/10.22478/ufpb.2317-6032.2017v21n3.29619

Otter, J. A., \& French, G. L. (2009). Bacterial contamination on touch surfaces in the public transport system and in public areas of a hospital in London. Lett Appl Microbiol, 49(0), 803805.

Peng, Y., Qianting O., Dongxin, L., Ping, X., Ying, L., Xiaohua, Y. et al. (2015). Metro system in Guangzhou as a hazardous reservoir of methicillin-resistant Staphylococci: findings from a point-prevalence molecular epidemiologic study. Scientific Reports, 5, 16087. https://doi.org/10.1038/srep16087

Pereira, J. N. P., Rabelo, M. A., Lima, J. L. C., Neto, A. M. B., Lopes, A. C. S., \& Maciel, M. A. V. (2016). Phenotypic and molecular characterization of resistance to macrolides, lincosamides and type B streptogramin of clinical isolates of Staphylococcus spp. of a university hospital in Recife, Pernambuco, Brazil. Brazilian Journal of Infectious Diseases, 20(3), 276-281. https://dx.doi.org/10.1016/j.bjid.2016.03.003

Pinheiro, T. R., \& Stopiglia, C. D. O. (2015). Potencial infeccioso do transporte coletivo universitário da cidade de Uruguaiana-RS. Anais do VII Salão Internacional de Ensino, Pesquisa e Extensão - Universidade Federal do Pampa, 7(2), 5621.

Santiago-Neto W., Machado G., Paim D. S., Campos T., Brito M. A. V. P., Cardoso

Santos, A. L., Santos, D. O., Freitas, C. C., Ferreira, B. L. A., Afonso, I. F. \& Rodrigues, C. R. et al. (2007). Staphylococcus aureus: visitando uma cepa de importância hospitalar. Jornal Brasileiro de Patologia e Medicina Laboratorial, 43(6), 413-423. https://dx.doi.org/10.1590/S1676-24442007000600005

Simões, R. R., Aires-de-Sousa, M., Conceição, T., Antunes, F., Costa, P. M., \& Lencastre, H. (2011). High Prevalence of EMRSA-15 in Portuguese Public Buses: A Worrisome Finding. PLOS ONE, 6(3), 1-5.

Stuchi, A. G., Oliveira, C. H. A. S., Soares, B. M., \& Arreguy-Sena, C. (2013). Contaminação bacteriana e fúngica dos telefones celulares da equipe de saúde em um hospital em Minas Gerais. Ciênc. Cuid. Saúde, 12(4), 760-767.

Tavares, W. (2000). Bactérias gram-positivas problemas: resistência do estafilococo, do enterococo e do pneumococo aos antimicrobianos. Rev Soc Bras Med Trop, 33(3), 281-301.

Winn, W.C., Allen, S.D., Janda, W.M., Koneman, E.W., Procop, G.W. et al. (2008). Koneman's color atlas and textbook of diagnostic microbiology (6th ed.). Rio de Janeiro: Guanabara Koogan, p. 617-665.

Yeh, P. J., Simon, D. M., Millar, J. A., Alexander, H. F. \& Franklin, D. (2011). A diversity of Antibiotic-resistant Staphylococcus spp. in a Public Transportation System. Osong Public Health and Research Perspectives. 2(3),202-209. 